\title{
Estudo da função muscular peribucal, do grau de inclinação vestíbulo-lingual e da discrepância de modelo dos incisivos inferiores permanentes em crianças respiradoras nasais e bucais com oclusão normal e má oclusão de Classe I
}

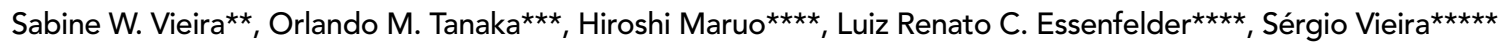

\begin{abstract}
Resumo
O objetivo deste trabalho foi verificar a existência de correlação entre o modo respiratório bucal, o comportamento dos músculos orbicular inferior da boca e mentoniano e a determinação de características oclusais específicas, na região anterior do arco dentário inferior. A amostra constituiu-se de 88 crianças brasileiras, leucodermas, sendo 49 do gênero masculino e 39 do gênero feminino, com média de idade de 6 anos e 11 meses, dividida em 4 subgrupos, portadores de: oclusão normal e modo respiratório nasal (ONRN); oclusão normal e modo respiratório bucal (ONRB); má oclusão de Classe I e modo respiratório nasal (CLIRN) e má oclusão de Classe I e modo respiratório bucal (CLIRB). Foram realizados 22 testes eletromiográficos, avaliação do grau de inclinação vestíbulo-lingual (FMIA) e da discrepância de modelo (DM) dos incisivos inferiores permanentes. Após terem sido submetidos à análise estatística os resultados indicaram que, quando comparados os subgrupos, não houve diferença estatisticamente significativa para as variáveis dentárias e para a maioria das variáveis eletromiográficas avaliadas. Desta forma, a alteração do modo respiratório não pôde ser relacionada às características oclusais e aos comportamentos musculares estudados.
\end{abstract}

Palavras-chave: Eletromiografia. Respiração bucal. Musculatura peribucal. Inclinação dentária.

\section{INTRODUÇÃO E REVISÃO DE LITERATURA}

Diversas foram as teorias elaboradas ao longo do tempo na tentativa de explicar a possível existência de uma relação de causa-efeito entre a manifestação do modo respiratório alterado (respiração bucal), a disfunção muscular peribucal e a alteração de determinadas características dentofaciais.

Alusões ao possível significado das forças musculares lábio-linguais, no estabelecimento das más

\footnotetext{
* Resumo de Dissertação apresentada ao Programa de Pós-Graduação em Odontologia da Pontifícia Universidade Católica do Paraná, Centro de Ciências Biológicas e da Saúde, como parte dos requisitos para a obtenção do Título de Mestre em Odontologia (Ortodontia).

** Mestre em Odontologia (Ortodontia). Pontifícia Universidade Católica do Paraná.

*** Professor Doutor em Odontologia (Ortodontia) - UFRJ.

Professor Doutor em Odontologia (Ortodontia) - UNICAMP.

Professor Doutor em Odontologia (Dentística) - FOB-USP.
} 
oclusões, surgiram anteriormente ao ano de 1873 e, já naquela época, Tomes ${ }^{43}$ afirmou que as forças musculares desenvolvidas durante os movimentos fisiológicos, principalmente pela língua e pelos lábios, seriam suficientes para determinar as posições dos dentes nos arcos dentários. Também neste sentido, Ballard ${ }^{4}$ mencionava que os lábios moldariam a forma dos arcos dentários, teoria esta também sustentada por Ballard ${ }^{5}$. Por outro lado, $\operatorname{Scott}^{37}$ e Hovel1 ${ }^{21}$ relataram não acreditar na existência de uma relação entre a forma do arco dentário e o comportamento dos tecidos moles.

Para a elucidação do questionamento quanto à consideração ou não da função muscular peribucal na determinação do posicionamento dentário, há que se considerar a notória escassez de subsídios no âmbito da Ortodontia. Apesar da introdução da eletromiografia na pesquisa ortodôntica por Moyers $^{31}$, metodologia esta adequada para a realização do diagnóstico da função muscular, suficientemente testada e já bastante utilizada por alguns autores $^{12,13,29,30,32,36,42,44,48}$, esta avaliação atualmente ainda é realizada sem qualquer fundamentação científica, levando ao uso de uma classificação genuinamente subjetiva dos lábios do paciente.

A respiração bucal (RB) é, dentre outras, a alteração ambiental que mais comumente atua sobre esse sistema e, talvez, a que produza as conseqüências mais severas. Estima-se que 85\% de todas as crianças apresentam algum grau de insuficiência nasal e que 20\% respiram habitualmente pela boca ${ }^{19,20}$.

Nesse particular, até que ponto a disfunção respiratória contribuiria para o estabelecimento das alterações dentofaciais geralmente a ela associadas? Atuaria a RB como causa ou efeito nesta relação? Quais seriam as suas prováveis conseqüências? Uma grande incerteza a respeito da existência e da natureza desta possível inter-relação ainda persiste. Propõe-se, então, determinar a existência de: eventuais influências da respiração bucal e/ou padrão oclusal sobre a função dos músculos orbicular inferior da boca e mentoniano; correlação entre a respiração bucal, o comportamento dos músculos orbicular inferior da boca e mentoniano, a inclinação vestíbulo-lingual e a discrepância de modelo dos incisivos inferiores permanentes.

\section{MATERIAL E MÉTODO}

Por meio de um levantamento populacional realizado em crianças cursando a $1^{\text {a }}$ série do ensino fundamental na rede estadual de ensino público da cidade de Curitiba e Região Metropolitana, selecionou-se uma amostra aleatória composta de crianças portadoras de "oclusão normal" (33 indivíduos) e crianças portadoras de característcas clínicas de má oclusão de Classe I (55 indivíduos), apresentando modo respiratório nasal ou bucal, do gênero feminino ou masculino, brasileiras, leucodermas, cujas idades variavam entre 6 anos e 1 mês e 8 anos e 2 meses.

Foram considerados como portadores de "oclusão normal" os indivíduos que apresentaram, em máxima intercuspidação habitual, características clínicas similares às propostas por Angle ${ }^{1}$ : relação vestíbulolingual normal entre os arcos dentários, linhas médias coincidentes, degrau mesial ou plano terminal reto dos segundos molares decíduos, relação dos caninos de Classe I e ausência de apinhamentos.

O diagnóstico do modo respiratório foi realizado por meio de critérios essencialmente clínicos, conforme proposto por Wagnitz ${ }^{47}$. Foram consideradas como respiradoras nasais, 32 crianças que respiraram pelo nariz durante o exame visual, mantendo o selamento labial quando na posição de repouso, sem qualquer esforço além do fisiológico. Além disto, nestes casos, não havia relato de respiração pela boca, durante o dia e à noite, e de haver ronco e baba noturna. Por outro lado, foram classificadas como respiradoras bucais 56 crianças que não apresentaram selamento labial espontâneo e satisfatório durante o exame anteriormente mencionado e/ou cujos relatos acusaram indícios de $\mathrm{RB}$, tais como: selamento labial intermitente, ronco e/ou baba noturna, quadros alérgicos recorrentes, etc. Por meio de exame otorrinológico encontrou-se, nestas crianças, 
a existência de obstruções ou alterações das vias aéreas superiores (amígdalas, septo, cornetos e adenóides) e/ou, pelo exame fonoaudiológico observou-se alguma disfunção na fonação e mastigação.

A amostra ficou então organizada em 4 subgrupos: ONRN (portadores de oclusão normal e modo respiratório nasal); ONRB (portadores oclusão normal e modo respiratório bucal); CLIRN (portadores de má oclusão de Classe I e modo respiratório nasal) e CLIRB (portadores de má oclusão de Classe I e modo respiratório bucal).

Foram feitas tomadas radiográficas em norma lateral, em máxima intercuspidação habitual e com os lábios em repouso, e realizados traçados utilizando-se transferidor cefalométrico, aproximando-se os valores obtidos para o 0,5 grau mais próximo. A precisão e a confiabilidade dos valores obtidos foram atestadas verificando-se a porcentagem do erro das variáveis, que não excedeu 3\% em relação às variâncias totais apresentadas pela amostra e, portanto, o método de mensuração foi considerado adequado.

Para a avaliação do grau de inclinação vestíbulo-lingual dos incisivos inferiores permanentes foi utilizada a medida cefalométrica angular FMIA ${ }^{45}$, que mede o ângulo entre o Plano de Frankfurt (passando pelos pontos cefalométricos Pório e Orbital) e o prolongamento do longo eixo do incisivo central inferior permanente. Para o cálculo da discrepância de modelo dos quatro incisivos inferiores permanentes foi utilizada a medida linear DM (discrepância de modelo).

A avaliação eletromiográfica do músculo orbicular inferior da boca, região medial (OI) e do músculo mentoniano (M) foi efetuada por um único operador, utilizando-se um eletromiógrafo de oito canais (Eletromiográfo MYO-TRONICS INC. Seattle, Washington, USA. Computador com processador INTEL - Pentium $120 \mathrm{MHz}$ ), calibrado em $100 \mu \mathrm{V}$, quando o músculo se encontrava na situação de repouso e em $500 \mu \mathrm{V}$, durante os movimentos predeterminados.

Foram utilizados eletrodos de superfície, na re- gião do músculo a ser estudado e eletrodos-terra, fixados no tórax do paciente, a fim de evitar eventuais interferências.

A seleção dos movimentos subordinou-se a dois aspectos fundamentais: movimentos que refletissem a atividade diária dos indivíduos e aqueles que permitissem melhor discriminação entre os subgrupos de indivíduos estudados. Foi analisada, primeiramente, a situação de repouso e, em seguida, obedeceu-se rigorosamente à ordem de simulações dos movimentos específicos previamente determinados, a saber: soprar com as bochechas flácidas (SFL); soprar com as bochechas distendidas(SDIS); sucção livre (SL); compressão recíproca dos lábios (CL); compressão dos lábios contra os dentes (CLD); afastamento das comissuras labiais (AC); projeção labial (PL); abertura máxima da boca (AM); pronúncia do fonema /bê/ (BE); pronúncia do fonema /ême/ (EME); pronúncia do fonema /pê/ (PE); pronúncia do fonema /éfe/ (EFE); pronúncia do fonema /vê/ (VE); protrusão mandibular (PRO); mastigação posterior direita de elástico ortodôntico (MORELLI, tamanho 5/16") (MD); mastigação posterior esquerda de elástico ortodôntico (ME); apertamento posterior direito de elástico ortodôntico (APERDM); apertamento posterior esquerdo de elástico ortodôntico (APEREM); lateralidade mandibular direita (LD); lateralidade mandibular esquerda (LE) e deglutição de saliva (DEG).

\section{RESULTADOS E DISCUSSÃO}

A análise estatística empregada constituiu-se do agrupamento de variáveis por histogramas de freqüência, análise dos coeficinetes de correlação, diagramas de dispensão, análise de variância e teste de Tuckey para a comparação das médias, com nível de significância de $5 \%$ de probabilidade.

Segundo a análise da variância, com as variáveis agrupadas pelo modo respiratório e oclusão dentária apresentada (MRO), foi possível constatar-se que não houve diferença estatística significante no comportamento do OI e M, 


\begin{tabular}{|c|c|c|c|c|c|c|c|c|}
\hline & Efeito & & & Erro & & & & \\
\hline & so & GL & $\mathbf{O M}$ & So & GL & $\mathbf{O M}$ & $\mathbf{F}$ & $\mathbf{p}$ \\
\hline FMIA & 63,055 & 3 & 21,018 & 4318,343 & 84 & 51,409 & 0,409 & 0,747 \\
\hline DM & 19,385 & 3 & 6,462 & 154,664 & 32 & 4,833 & 1,337 & 0,280 \\
\hline REPOI & 227,780 & 3 & 75,927 & 20124,364 & 83 & 242,462 & 0,313 & 0,816 \\
\hline REPM & 323,050 & 3 & 107,683 & 16050,159 & 84 & 191,073 & 0,564 & 0,641 \\
\hline SFOI & 1277,710 & 3 & 425,903 & 27260,484 & 84 & 324,530 & 1,312 & 0,276 \\
\hline SFM & 337,017 & 3 & 112,339 & 17786,372 & 84 & 211,743 & 0,531 & 0,663 \\
\hline SDISOI & 1204,508 & 3 & 401,503 & 26670,088 & 84 & 317,501 & 1,265 & 0,292 \\
\hline SDISM & 1449,339 & 3 & 483,113 & 26901,656 & 84 & 320,258 & 1,509 & 0,218 \\
\hline SLOI & 695,371 & 3 & 231,790 & 23085,414 & 84 & 274,826 & 0,843 & 0,474 \\
\hline SLM & 513,636 & 3 & 171,212 & 17002,991 & 84 & 202,417 & 0,846 & 0,473 \\
\hline CLOI & 364,196 & 3 & 121,399 & 50319,003 & 84 & 599,036 & 0,203 & 0,894 \\
\hline CLM & 12639,413 & 3 & 4213,138 & 148469,082 & 84 & 1767,489 & 2,384 & 0,075 \\
\hline CLDOI & 271,914 & 3 & 90,638 & 84795,983 & 84 & 1009,476 & 0,090 & 0,965 \\
\hline CLDM & 6382,292 & 3 & 2127,431 & 103047,768 & 84 & 1226,759 & 1,734 & 0,166 \\
\hline ACOI & 3450,810 & 3 & 1150,270 & 53843,089 & 84 & 640,989 & 1,795 & 0,154 \\
\hline ACM $^{*}$ & 22025,373 & 3 & 7341,791 & 149750,518 & 84 & 1782,744 & 4,118 & $0,009 *$ \\
\hline PLOI & 3777,318 & 3 & 1259,106 & 152263,482 & 84 & 1812,660 & 0,695 & 0,558 \\
\hline PLM & 3143,439 & 3 & 1047,813 & 132649,629 & 84 & 1579,162 & 0,664 & 0,577 \\
\hline AMOI & 3940,916 & 3 & 1313,639 & 172089,807 & 84 & 2048,688 & 0,641 & 0,591 \\
\hline AMM & 10486,782 & 3 & 3495,594 & 248470,317 & 84 & 2957,980 & 1,182 & 0,322 \\
\hline BEOI & 7137,908 & 3 & 2379,303 & 92545,473 & 84 & 1101,732 & 2,160 & 0,099 \\
\hline BEM & 3323,067 & 3 & 1107,689 & 118326,376 & 84 & 1408,647 & 0,786 & 0,505 \\
\hline EMEOI & 3769,857 & 3 & 1256,619 & 61656,396 & 84 & 734,005 & 1,712 & 0,171 \\
\hline EMEM & 3622,260 & 3 & 1207,420 & 93006,051 & 84 & 1107,215 & 1,091 & 0,358 \\
\hline PEOI & 2180,141 & 3 & 726,714 & 71239,145 & 84 & 848,085 & 0,857 & 0,467 \\
\hline PEM & 2279,957 & 3 & 759,986 & 103389,630 & 84 & 1230,829 & 0,617 & 0,606 \\
\hline EFEOI & 3284,524 & 3 & 1094,841 & 85683,812 & 83 & 1032,335 & 1,061 & 0,370 \\
\hline EFEM & 1496,416 & 3 & 498,805 & 64996,459 & 83 & 783,090 & 0,637 & 0,593 \\
\hline VEOI & 663,806 & 3 & 221,269 & 61546,631 & 84 & 732,698 & 0,302 & 0,824 \\
\hline VEM & 389,313 & 3 & 129,771 & 73405,808 & 84 & 873,879 & 0,148 & 0,930 \\
\hline PROOI & 422,945 & 3 & 140,982 & 93889,795 & 83 & 1131,202 & 0,125 & 0,945 \\
\hline PROM & 629,672 & 3 & 209,891 & 150306,015 & 83 & 1810,916 & 0,116 & 0,951 \\
\hline LDOI & 2150,501 & 3 & 716,834 & 72839,169 & 84 & 867,133 & 0,827 & 0,483 \\
\hline LDM & 1876,070 & 3 & 625,357 & 56048,112 & 84 & 667,239 & 0,937 & 0,426 \\
\hline LEOI & 1211,291 & 3 & 403,764 & 41042,181 & 84 & 488,597 & 0,826 & 0,483 \\
\hline LEM & 197,866 & 3 & 65,955 & 43561,007 & 84 & 518,583 & 0,127 & 0,944 \\
\hline MDOI* & 8153,021 & 3 & 2717,674 & 82479,334 & 84 & 981,897 & 2,768 & $0,047^{*}$ \\
\hline MDM & 1083,034 & 3 & 361,011 & 43009,965 & 84 & 512,023 & 0,705 & 0,552 \\
\hline MEOI & 7788,503 & 3 & 2596,168 & 101125,053 & 84 & 1203,870 & 2,157 & 0,099 \\
\hline MEM & 1686,163 & 3 & 562,054 & 61824,135 & 84 & 736,002 & 0,764 & 0,518 \\
\hline APERDOI & 6142,912 & 3 & 2047,637 & 92651,984 & 84 & 1103,000 & 1,856 & 0,143 \\
\hline APERDM & 1486,749 & 3 & 495,583 & 98750,383 & 84 & 1175,600 & 0,422 & 0,738 \\
\hline APEREOI & 2069,674 & 3 & 689,891 & 78200,489 & 84 & 930,958 & 0,741 & 0,531 \\
\hline APEREM & 1994,685 & 3 & 664,895 & 67408,744 & 84 & 802,485 & 0,829 & 0,482 \\
\hline DEGOI* & 7094,026 & 3 & 2364,675 & 67290,323 & 84 & 801,075 & 2,952 & $0,037^{*}$ \\
\hline DEGM & 3005,546 & 3 & 1001,849 & 43527,518 & 84 & 518,185 & 1,933 & 0,130 \\
\hline
\end{tabular}

SQ - soma dos quadrados; GL - grau de liberdade; QM - quadrado médio; F - distribuição; p - probabilidade. A análise de variância foi realizada com o modelo inteiramente casualisado.

*Diferença estatística significante $(p<0,05)$ 
em todos os subgrupos estudados e na maioria dos testes realizados, inclusive para as variáveis que expressam a inclinação vestíbulo-lingual e a discrepância de modelo dos incisivos inferiores (Tab. 1), com exceção, apenas, para as variáveis ACM, MDOI e DEGOI, cujas médias apresentaram diferenças estatísticas significantes $(\mathrm{p}<0,05)$, o que pode ser atribuído à própria natureza do experimento que respeitou a individualidade das crianças estudadas.

Observa-se, portanto, que a grande maioria das variáveis obedece à distribuição normal, confirmando, assim, a hipótese de nulidade para esta análise, ou seja, de que as médias seriam estatisticamente iguais.

A hipótese de que a musculatura peribucal seria, dentre outros fatores, o elemento local que determinaria a forma dos contornos dos arcos dentários foi primeiramente considerada por Tomes em $1873^{43}$, quando relacionou as alterações da forma dos arcos dentários com a presença de tecidos hipertróficos localizados internamente nas bochechas.

A realização de estudos mais específicos, destinados a quantificar as pressões exercidas pelos tecidos moles sobre as estruturas dentárias adjacentes, alguns fatores que tradicionalmente vinham sendo aceitos como determinantes do posicionamento dentário, passaram a assumir um papel menos relevante. No entanto, verdadeiros avanços no estudo da possível influência das forças exercidas pelos tecidos moles na determinação do contorno dos arcos dentários apenas se tornaram possíveis com o advento de técnicas miométricas e eletromiográficas padronizadas, as quais passaram a ser utilizadas na pesquisa ortodôntica desde a metade deste século.

Moyers $^{31}$ introduziu a eletromiografia na pesquisa odontológica para o estudo da função muscular por meio da avaliação dos potenciais elétricos emanados pelos músculos, no momento de sua contração.

A determinação do padrão funcional dos músculos orbicular superior e inferior da boca, em indivíduos portadores de oclusão normal, foi a preocupação de Essenfelder ${ }^{12}$ quando estudou o comportamento destes músculos em indivíduos portadores de oclusão normal. Por outro lado, Simpson $^{39}$ demonstrou, entre outros achados, falta de correlação entre a atividade labial e os parâmetros esqueléticos e dentários. Essenfelder e Vitti ${ }^{12}$ encontraram ausência de atividade do músculo orbicular inferior durante a deglutição e o repouso. Estudando indivíduos com oclusão normal e indivíduos portadores de má oclusão de Classe I, antes e após o tratamento ortodôntico, Sales e Vitti ${ }^{36}$ concluíram que a má oclusão dentária exerceria influência na atividade da musculatura peribucal.

Após a análise eletromiográfica do músculo orbicular inferior da boca, Marchiori ${ }^{29}$ observou que não houve diferença estatística significativa para os indivíduos com má oclusão de Classe I, em relação aos portadores de oclusão normal, durante a execução dos movimentos lábio-mandibulares. Já Zilli ${ }^{46}$ observou maior atividade $\mathrm{OI}$, em indivíduos portadores de má oclusão de Classe I, se comparados a indivíduos com oclusão normal, durante os movimentos de emissão do fonema /ême/ e mastigação, se comparada à atividade exercida na posição de repouso.

Por meio deste trabalho, observou-se um comportamento muscular similar entre indivíduos com oclusão normal e indivíduos portadores de má oclusão de Classe I e ainda que indivíduos com a mesma função muscular apresentaram, também, valores estatisticamente iguais para as variáveis FMIA e DM, a despeito das diferentes características oclusais anteriores apresentadas pelos indivíduos portadores de má oclusão de Classe I, aqui estudados.

Portanto, as suspeitas clínicas a respeito da participação do ambiente muscular que envolve a dentição no posicionamento dentário, levantadas por Tomes (1873 apud COOPER ${ }^{11}$ ); Rix $^{34,35}$; Strang ${ }^{41}$; Ballard $^{3,4,5}$; Gwynne-Evans ${ }^{15,16}$; Brodie ${ }^{9,10}$ e Ricket$\mathrm{ts}^{33}$, não puderam ser confirmadas por esta pesquisa.

Não houve correlação entre o comportamento muscular e as variáveis dentárias estudadas, o que confirma os resultados obtidos por Simpson ${ }^{39}$ 
e Lima ${ }^{23}$ porém diverge das opiniões de $\mathrm{Moller}^{30}$, Simpson ${ }^{40}$ e Harradine e Kirschen ${ }^{18}$, de que haveria maior atividade dos músculos em questão, quando em presença de incisivos inferiores retroinclinados, e das afirmações de Gustafsson e Ahlgren ${ }^{14}$ que observaram maior atividade elétrica do OI quando em presença de incisivos inferiores proclinados.

Há que se considerar, no entanto que, excetuando os trabalhos eletromiográficos de Gustafsson e Ahlgren ${ }^{14}$, Essenfelder ${ }^{12}$, Essenfelder e Vitti ${ }^{13}$, Sales e $\mathrm{Vitti}^{36}$ e Zilli ${ }^{48}$, as amostras estudadas pela maioria dos autores, continham, em sua maior parte, indivíduos portadores de outros tipos oclusais, associados a diferentes comprometimentos esqueléticos ântero-posteriores (má oclusão de Classe II, divisão 1 e 2), o que poderia, eventualmente, ter ocasionado a má relação anterior do arco dentário, permitindo a interposição física do lábio inferior entre os incisivos superiores e inferiores, o que também poderia resultar na retroinclinação dentária inferior, assim como já defendiam Brodie ${ }^{9}$, Ballard $^{4}$ e Gwynne-Evans ${ }^{16}$. Este fato poderia, efetivamente, influenciar sobremaneira os resultados por eles obtidos, caso não houvesse um tratamento específico para cada tipo oclusal apresentado.

A isenção da musculatura estudada de exercer influências na determinação do grau de inclinação vestíbulo-lingual e do grau de discrepância de modelo apresentados pelos incisivos inferiores, em última análise, não sustenta as hipóteses de que a função muscular, pelo menos o momento do crescimento e desenvolvimento físico do indivíduos estudados, seria um elemento causal primário ou secundário na determinação da posição espacial dos dentes. Por outro lado, há que se considerar que, na hipótese de ter havido alguma alteração no posicionamento dos incisivos inferiores, provocada pela musculatura peribucal adjacente, poderia também, em contrapartida, ter havido a devida adaptação muscular a este outro posicionamento dentário assumido, o que, em última análise, justificaria a ausência de diferenças significativas na atividade muscular verificada para os subgrupos.
No entanto, a semelhança da função muscular do OI verificada, em indivíduos portadores de má oclusão de Classe I e em indivíduos com oclusão normal, também observada por Marchiori ${ }^{29}$, não confirma os resultados de Sales e Vitti ${ }^{36}$ e Zilli ${ }^{46}$ que observaram diferença estatística significativa na atividade eletromiográfica deste músculo, entre indivíduos com os tipos oclusais anteriormente mencionados. Este fato poderia também significar uma acomodação dos tecidos moles diante das características oclusais apresentadas.

Paralelamente, o possivel efeito da função respiratória sobre as estruturas dentofaciais igualmente gerava significativa controvérsia no meio ortodôntico e, nesta perspectiva, diversos autores, apoiados na experiência clínica adquirida, procuraram relacionar a alteração da função respiratória nasal às estruturas do complexo craniofacial.

Segundo a metodologia e a faixa etária empregadas, a alteração do modo respiratório igualmente não acarretou diferentes comportamentos dos músculos peribucais, nem diferentes características oclusais para os incisivos inferiores (FMIA e DM). Da mesma forma como afirmaram Gwynne-Evans e Ballard ${ }^{17}$, Leech ${ }^{22}$, Linder-Aronson e Bäckström ${ }^{28}$ e Lima $^{23}$ não foi possível estabelecer correlações entre as variáveis cefalométrica (FMIA), dentária (DM) e eletromiográficas com o modo respiratório ou tipo oclusal apresentados.

Os resultados aqui obtidos não confirmaram as suspeitas clínicas levantadas por Robert e Tomes (1873 apud COOPER ${ }^{11}$ ); Angle $^{1}$ e Ricketts ${ }^{33}$, quando sugeriram que, por influência direta da $\mathrm{RB}$, indivíduos respiradores bucais (RB) apresentariam maior inclinação vestibular dos incisivos inferiores. Igualmente, as conclusões a partir dos experimentos de Linder-Aronson ${ }^{24,25,26,27}$, Bresolin et al. ${ }^{8}$, e Behlfelt et al. ${ }^{6,7}$, alegando que os indivíduos $\mathrm{RB}$ apresentariam incisivos inferiores mais retroinclinados, não puderam ser confirmadas pelos presentes resultados.

Os dados eletromiográficos aqui verificados são também conflitantes com as opiniões de Moller ${ }^{30}$, 
Gustaffson e Ahlgren ${ }^{14}$, Harradine e Kirschen ${ }^{18}$, Tosello $^{44}$, Pallú et al. ${ }^{32}$, Tomé e Marchiori' ${ }^{42}$ e Silva et $\mathrm{al}^{38}$, que relacionavam a RB à determinação de disfunções nos tecidos musculares labiais que, por sua vez, poderiam levar ao estabelecimento de maus posicionamentos dentários. Talvez esta divergência entre resultados tenha sido decorrente dos diferentes critérios considerados para o diagnóstico do modo respiratório, utilizando amostras com diferentes comprometimentos respiratórios, fato que prejudicaria o estabelecimento de comparações.

Em idade mais precoce, como é o caso da presente amostra, a menor maturação da função muscular peribucal, o menor crescimento e menor estágio de desenvolvimento facial e nasal, o maior desenvolvimento dos tecidos linfóides e o menor tempo de atuação do hábito de RB, caso existente, poderiam representar diferentes comprometimentos respiratórios dos indivíduos estudados. Tal variabilidade pode ter contribuído, eventualmente, para os diferentes resultados aqui obtidos em relação àqueles dos demais trabalhos que estabeleceram comparações entre indivíduos portadores de RB e RN.

Existe, contudo, a possibilidade das possíveis alterações neuromusculares e dentárias, oriundas do modo respiratório alterado, ainda não terem se manifestado em idade precoce. É importante considerar também que, na amostra em questão, houve o predomínio da harmonia no estabelecimento do padrão facial, favorecendo o bom relacionamento ântero-posterior dos arcos dentários e, eventualmente, oferecendo, ao mesmo tempo, maior resistência para as possivieis manifestações provocadas pelo modo respiratório bucal. Havendo harmonia nos padrões faciais, a função muscular também poderá se encontrar-se em equilíbrio, oferecendo resistência ao estabelecimento de disfunções neuromusculares decorrentes da alteração do modo respiratório.

Também nesse sentido, observa-se a existência de especulações na tentativa de classificar os lábios dos indivíduos RB em hipo/normo/hipertônicos ou hipo/normo/hiperativos ${ }^{25,28}$ e em competentes ou incompetentes ${ }^{14,18,39,40,44}$. Da mesma forma, acreditando que o padrão morfo-funcional dos lábios seria determinante de características oclusais, Brodie $^{9,10}$ e Ricketts ${ }^{33}$ acreditavam que lábios mais finos desenvolveriam maior pressão sobre a dentição adjacente, levando, desta maneira, ao estabelecimento de um maior apinhamento dentário anterior, o que já não ocorreria em presença de lábios mais espessos.

É importante ressaltar que a confiabilidade em tais interpretações carece ainda de comprovação científica. Pelo menos no que diz respeito ao posicionamento espacial dos lábios, o presente trabalho não encontrou diferenças significativas na função muscular entre os subgrupos dos indivíduos estudados, a despeito destes apresentarem ausência ou presença de selamento labial, impedindo, assim, a utilização do mesmo como evidência clínica da atividade labial desenvolvida.

Percebe-se, dessa maneira, que determinados conceitos antigos, tão enraizados no pensamento ortodôntico, passam, então, a merecerem alguns questionamentos pois, pelo menos na faixa etária e metodologia consideradas, a RB não apresentou qualquer influência sobre a função muscular e sobre a determinação das características oclusais aqui avaliadas.

\section{CONCLUSÃO}

Com base nos resultados obtidos pôde-se concluir que, segundo a metodologia aqui empregada e na faixa etária envolvida:

1) não há diferença entre a função muscular de indivíduos respiradores bucais, indivíduos respiradores nasais, indivíduos portadores de má oclusão de Classe I e indivíduos com oclusão normal;

2) não há correlação entre a respiração bucal, a função muscular peribucal, o grau de inclinação vestíbulo-lingual e a discrepância de modelo dos incisivos inferiores.

Enviado em: Maio de 2003 Revisado e aceito: Setembro de 2003 


\title{
A perioral muscles activity, buccal-lingual inclination and space discrepancy of the lower incisors study, in mouth or nasal breathing children with normal or Class I malocclusion
}

\begin{abstract}
The purpose of this study was to verify the existence of any correlation between the mouth breathing, the inferior orbicularis oris and mentalis muscles activity and specific dental features in the anterior area of the lower dental arch. A sample formed by 88 leucodermas brazilian children, 49 boys and 39 girls, with a medium age of 6 years and 11 months, was divided into 4 subgroups according to the oclusal type and breathing mode presented: normal occlusion and nasal breathing (ONRN), normal occlusion and mouth breathing (ONRB), Class I malocclusion and nasal breathing (CLIRN) and Class I malocclusion and mouth breathing (CLIRB). This research involved the performance of 22 electromyographic tests, the quantitative assessment of the buccal-lingual inclination (FMIA) and space discrepancy (DM) of the permanent lower incisors. Obtained results were submitted to statistic methods and showed no significant statistical difference for the dental variables and for the majority of the electromyographic variables studied. In this way, the clinically assessed mouth breathing could not be associated to the oclusal features and muscular functions studied.
\end{abstract}

Key words: Electromyography. Mouth breathing. Perioral muscles. Dental inclination.

\section{REFERÊNCIAS}

1. ANGLE, E. H. Classification of malocclusion. Dent Cosmos, Philadelphia, v. 41, n. 3, p. 248-64, 1899

2. ANGLE, E. H. Treatment of malocclusion of the teeth. 7. ed Philadelphia: S. S. White, 1907.

3. BALLARD, C. F. Some bases for aetiology and diagnosis in Orthodontics. Dent Rec, London, v. 68, no. 6, p. 133-145, June 1948.

4. Factors of skeletal and soft tissue morphology affecting prognosis and treatment planning in orthodontics. Dent J Aust Sydney, v. 25, p. 254-264, Nov./Dec. 1953.

5. The clinical significance of innate and adaptive postures and motor behaviour. Dent Pract, Bristol, v. 12, no. 6, p. 219 228, Feb. 1962

6. BEHLFELT, K.; LINDER-ARONSON, S.; McWILLIAM, J. et al. Dentition in children with enlarged tonsils compared to control children. Eur J Orthod, London, v. 11, no. 4, p. 416-429, 1989.

7. Cranio-facial morphology in children with and without enlarged tonsils. Eur J Orthod, London, v. 12, p. 233-243, 1990.

8. BRESOLIN, D.; SHAPIRO, P. A.; SHAPIRO, G. G. et al. Mouth breathing in allergic children: its relationship to dentofacial development. Am J Orthod, St. Louis, v. 83, no. 4, p. 334-340, Apr. 1983.

9. BRODIE, A. G. Considerations of musculature in diagnosis, treatment and retention. Am J Orthod, St. Louis, v. 38, p. 823-835, 1952.

10. Muscular factors in the diagnosis and treatment of maloc clusions. Angle Orthod, Appleton, v. 23, no. 2, p. 71-77, Apr. 1953.

11. COOPER, B. C. Nasorespiratory function and orofacial development. Otolaryngol Clin North Am, Philadelphia, v. 22, no. 2, p. 413-441, Apr. 1989.

12. ESSENFELDER, L. R. C. Análise eletromiográfica dos músculos orbicularis em jovens portadores de oclusão normal.
1975. Dissertação (Mestrado) - UNICAMP, Piracicaba, 1975.

13. ESSENFELDER, L. R. C.; VITTI, M. Análise eletromiográfica dos músculos orbicularis oris em jovens portadores de oclusão normal. Ortodontia, São Paulo, v. 10, n. 3, p. 180-191, set./dez. 1977.

14. GUSTAFSSON, M.; AHLGREN, J. Mentalis and orbicularis oris activity in children with incompetent lips. Acta Odontol Scand, Stockholm, v. 33, p. 355-363, 1975.

15. GWYNNE-EVANS, E. The organization of the orofacial muscles in relation to breathing and feeding. Br Dent J, London, v. 91, no. 6, p. 135-142, Sept. 1951.

16. __ An analysis of the orofacial structures with special reference to muscle behaviour and dental alignment. Am J Orthod St. Louis, v. 40, p. 715-720, 1954. Abstracts and reviews.

17. GWYNNE-EVANS, E.; BALLARD, C. F. Discussion on the mouth-breather. Proc R Soc Méd, London, v. 51, p. 279-285, Oct. 1957.

18. HARRADINE, N. W. T.; KIRSCHEN, R. H. E. S. Lip and mentalis activity and its influence on incisor position - a quantitative electromyographic study. Br J Orthod, London, v. 10, no. 3 , p. 114-127, July 1983.

19. HAWKINS, A. C. Mouth breathing as the cause of malocclusion and other facial abnormalities. Tex Dent J, Dallas, v. 83, p. 1015, June 1965.

$20 . \quad$ Mouth breathing and its relationship to malocclusion and facial abnormalities. N M Dent J, Albuquerque, no. 8, p. 18-21, May 1969.

21. HOVELL, J. H. The relationship of the oro-facial musculature to occlusion: current British thought. In: KRAUS, B. S.; RIEDEL, R. Vistas in Orthodontics, Philadelphia: Lea \& Febiger, 1962, p. 328-345.

22. LEECH, H. L. A clinical analysis of orofacial morphology and behaviour of 500 patients attending an upper respiratory research clinic. Dent Pract, Ewell, v. 9, no. 4, p. 57-68, Dec. 1958. 
23. LIMA, M. H. Estudo morfofuncional dento-muscular em indivíduos com oclusão normal e maloclusão Classe II, divisão 1, que apresentam modos respiratórios predominantemente nasal e bucal. 2002. Dissertação (Mestrado) - PUCPR, Curitiba, 2002.

24. LINDER-ARONSON, S. Adenoids. Their effect on mode of breathing and nasal airflow and their relationship to characteristics of the facial skeleton and the dentition. Acta Otolaryngol, Stockholm, v. 265, p. 1-132, 1970. Suppl.

25. Effects of adenoidectomy on dentition and nasopharynx. Am J Orthod, St. Louis, v. 65, no. 1, p. 1-15, Jan. 1974.

26. Respiratory function in relation to facial morphology and the dentition. Br J Orthod, London, v. 6, p. 59-71, 1979a.

27. Naso-respiratory function and craniofacial growth. In: Mc NAMARA Jr., A. J. Naso-respiratory function and craniofacial growth. Ann Arbor: Center of Human and Development, University of Michigan, 1979b., Craniofacial Growth Series, Monograph 9.

28. LINDER-ARONSON, S. BÄCKSTRÖM, A. A comparison between mouth and nose breathers with respect to occlusion and facial dimensions. Odontol Revy, Lund, v. 11, no. 4, p. 343-376, 1960.

29. MARCHIORI S. C Análise eletromiográfica do músculo orbicular da boca em indivíduos com oclusão normal e com maloclusões Classes I, II divisão 1 e III de Angle. 1993. Tese (Doutorado) - Universidade Estadual Paulista de Botucatu, Botucatu, 1993.

30. MOLLER, E. The chewing apparatus. Acta Physiol Scand, Stockholm, v. 280, no. 69, p. 9-229, 1966. Suppl.

31. MOYERS, R. E. Temporomandibular muscle contraction patterns in Angle Class II, division 1 malocclusions: an electromyographic analysis. Am J Orthod, St. Louis, v. 35, no. 11, p. 837-857, Nov. 1949

32. PALLÚ, V. R.; MAGNANI, M. B. B. A.; BERZIN, F.; BEVILAQUA D. Alterações musculares em indivíduos respiradores bucais. Publicatio UEPG Ponta Grossa, v. 2, n. 1, p. 73-89, 1996.

33. RICKETTS, R. M. Esthetics, environment, and the law of lip relation. Am J Orthod, St. Louis, v. 54, no. 4, p. 272-289, Apr. 1968a.

34. RIX, R. E. Deglutition and the teeth. Dent Rec, London, v. 66, no. 5 , p. 103-108, May 1946

35. Some observations upon the environment of the incisors. Dent Rec, London, v. 73, p. 427-441, Apr. 1953.
36. SALES, R. D.; VITTI, M. Análise eletromiográfica dos músculos orbicularis oris em indivíduos portadores de má-oclusão Classe I, antes e após submetidos a tratamento ortodôntico. Rev Ass Paul Cirurg Dent, São Paulo, v. 33, n. 5, p. 399-410, set. 1979.

37. SCOTT, J. The role of the soft tissues in determining normal and abnormal dental occlusion. Dent Practnr, Ewell, v. 11, no. 9, p. 302-308, May 1961.

38. SILVA, A. M. T.; MARCHIORI, S. C.; RIBEIRO, E. C.; CECHELLA C. Electromyography: evaluation of orbicularis oris muscles in rest and maximum contraction in mouth breathers children, before and after myotherapy. [S.I.]: ISEK, 2002.

39. SIMPSON, M. M. Lip incompetence and its relationship to skeletal and dental morphology - an electromyographic investigation. Br J Orthod, London, v. 3, no. 3, p. 177-179, 1976.

40. An electromyographic investigation of the perioral musculature in Class II division 1 malocclusion. $\mathbf{B r} \mathbf{J}$ Orthod London, v. 4, no. 1, p. 17-22, 1977.

41. STRANG, R. H. W. The fallacy of denture expansion as a treatment. J Clin Orthod, Boulder, v. 19, no. 1, p. 12-22, 1949

42. TOMÉ, M. C.; MARCHIORI, S. C. Estudo eletromiográfico dos músculos orbiculares superior e inferior da boca em crianças respiradoras nasais e bucais durante o repouso com e sem contato labial. JBO, Curitiba, v. 3, n. 5, p. 59-66, maio/jun. 1998.

43. TOMES, C.S. The bearing of the development of the jaws on rregularities. Dent Cosmos, Philadelphia, v.15, p.292-6, 1873

44. TOSELLO, D. O. Análise eletromiográfica dos músculos orbicular da boca e mentoniano, em indivíduos portadores de maloclusão Classe II divisão 1 de Angle. 1995. Tese (Doutorado) - UNICAMP, Piracicaba, 1995

45. TWEED, C. H. The Frankfort-mandibular incisor angle (FMIA) in orthodontic diagnosis, treatment planning and prognosis. Angle Orthod, Appleton, v. 24, no. 3, p. 121-77, July 1954.

46. VIEIRA, S. Introdução à bioestatística. 5. ed. Rio de Janeiro: Ed. Campus, 1988.

47. ZILLI, A. S. Estudo eletromiográfico dos músculos orbiculares da boca, segmentos superior e inferior (região medial), em jovens com maloclusão Classe I de Angle. 1994. Dissertação (Mestrado) - UNICAMP, Piracicaba, São Paulo.

48. WAGNITZ, S. J. V. Avaliação do grau de confiabilidade do diagnóstico clínico do modo respiratório bucal. 2000. Dissertação (Mestrado) - PUCPR, Curitiba, Paraná.
Endereço para correspondência

Sabine Westphal Vieira

Rua Martim Afonso, 779 - Apto. 501

CEP: $80430-100$

Curitiba-PR

E-mail: sabinevieira@terra.com.br 